\title{
COMPORTAMENTO DA Haematobia irritans EM FAZENDAS COM DIFERENTES MANEJOS DE BOVINOS
}

\author{
BEHAVIOUR Haematobia irritans IN HERD CATTLE FARMS \\ UNDER DIFFERENT MANAGEMENT
}

Gilson Pereira de Oliveira' ${ }^{1}$ Alfredo Ribeiro de Freitas ${ }^{1}$

\section{RESUMO}

A dinâmica parasitária da Haematobia irritans foi estudada em três fazendas com diferentes tipos de manejo de bovinos mestiços na região de São Carlos, SP, as quais não utilizavam nenhum tratamento especifico à mosca. $O$ experimento consistuiu na contagem de mosca na região dorso-lombar a cada 14 dias no periodo de outubro de 1992 a outubro de 1994. Cada fazenda adotava um tratamento: T, (Fazenda Ribeirão Bonito, verminose tratada com levamizole-Ripercol L injetável, carrapato com piretróide - Ectoplus "pour on" e berne com trichlorphonNeguvon + óleo queimado, uso tópico); $T_{2}$ (Fazenda São Carlos, verminose tratada com levamizole - Ripercol injetável, carrapato tratado com banho de imersão com formamidina - Triatox e berne com fenthion - Tiguvon "pour on") e $T_{3}$ (Fazenda Santa Eudóxia sem tratamento). Os dados de contagem da mosca-do-chifre (MC), na escala $\sqrt{M C \cdot 0,5}$, foram analisados em um modelo que incluiu além da média, os efeitos de tratamentos, ano, mês, periodo e as interações duplas, sendo que todos os efeitos diferiram entre si $(P \leq 0,01)$. Na escala original observou-se que a contagem nos meses de temperaturas mais elevadas (outubro a março), houve uma carga de 15,7+36,4 moscas/animal, enquanto que nos meses de temperaturas mais baixas (abril a setembro) foi de 6,8 $+23,6$ moscas/animal. A média de infestação de moscas/animal diferiu $(P \leq 0,01)$ entre os anos $(92=4,8 \pm 7,8 ; 93=8,8+20,0 ; 94=$ $16,6+43,8)$ e entre fazendas $\left(T_{1}=3,1+9,7 ; T_{2}=2,2 \pm 13,7 \mathrm{e}\right.$ $\left.T_{3}=26,0+44,5\right)$, mostrando que o comportamento da mosca-dochifre é irregular e aciclico. Observou-se ainda, uma eficiência $(P<0,05)$ de $81,1 \%$ e $86,3 \%$ nos tratamentos $T_{1}$ e $T_{2}$, respectivamente, quando comparado com o tratamento $T_{3}$.

Palavras-chave: bovino, mosca-do-chifre, comportamento, controle.

\section{SUMMARY}

Haematobia irritans parasitical dynamics was studied in three farms rearing crossbred beef cattle, under different management systems, in the region of São Carlos, São Paulo State. In the farms was not utilized any specific treatment against horn flies. The experiment consisted in counting fortnightly the number of flies on the dorso-lombar area of the animals, from October, 1992 to October, 1994. Each farm used a different parasite control: $T_{1}$ - Ribeirão Bonito Farm, anthihelminthic treatment, with injectable levamizole (Ripercol L); ticks treated with pour on pyrethroid compound (Ectoplus); and berne, treated with trichlorphon (Neguvon) plus used motor oil, applied topically; $T_{2}$ São Carlos Farm, antihelminthic treatmen with injectable levamizole (Ripercol L); ticks treated with formamidine (Triatox) in immersion bath; and berne treated with pour on fenthion (Tiguvon); and $T_{3}$ - Santa Eudóxia Farm, no parasitism treatments. The number of horn flies (MC) was analised in the scale $\sqrt{M C \cdot 0.5}$ in a statistical model that included, besides the mean, the effects of treatments, year, month, period and the double interactions. In the scale $M C$, all the effects differed from each other $(P \leq 0.01)$. During the months in which the temperature was higher (October to March) average parasite load was of $15.7 \pm 36.4$ flies, while temperature was lower (April to August) it was of $6.8+23.6$ flies. The average parasite load differed $(P \leq 0.01)$ among years $(92$ $=4.8 \pm 7.8 ; 93=8.8+20.0 ; 94=16.6 \pm 43.8$ ) and among farms $\left(T_{1}=3.1 \pm 9.7 ; T_{2}=2.2 \pm 13.7\right.$ e $\left.T_{3}=26.0 \pm 44.5\right)$, indicating that horn fly behaviour is irregular and noncyclic. The efficiency of $81.1 \%$ and $86.3 \%$ was observed for treatment $T_{1}$ and $T_{2}$ respectivelly, when compared to treatment $T_{3}$.

Key words: herd cattle, horn fly, behaviour, control. 'Pesquisador Dsc. da EMBRAPA - Centro de Pesquisa de Pecuária do Sudeste, Caixa Postal 339, 13560-970, São Carlos, SP. Bolsista
do CNPq. OLIVEIRA é o autor para correspondência. 


\section{INTRODUÇÃO}

A Haematobia irritans diante de suas infestações nos bovinos, tem sido motivo de alarde pelos pecuaristas desde o início de sua dispersão em território brasileiro. Possivelmente, esta situação tenha existido, em parte devido ao despreparo dos criadores e também pela propaganda veiculada pelos laboratórios com o intuito de ganhar a competitividade dos mosquicidas lançados no mercado. Por outro lado, a interação dos nossos técnicos com a literatura estrangeira sobre o assunto, motivou precauções exageradas, culminando, desta forma, em controles inadequados.

Hoje, as propriedades rurais convivem mais harmoniosamente com a presença da mosca, tomando decisões menos precipitadas. Acontece que uma série de informações à disposição do meio rural tornaram o homem do campo mais cauteloso sobre o assunto. Isso se deve, em parte, à continuidade dos trabalhos de pesquisa na área que vêm trazendo, cada vez mais, informações do comportamento da $\boldsymbol{H}$. irritans em nossas condições.

Nos Estados Unidos, onde o ectoparasitismo pela mosca-do-chifre se destaca com exclusividade nos bovinos, e assume situações críticas, a necessidade do envolvimento com inúmeros processos alternativos de controle, na tentativa de solucionar o problema, tornou-se uma condição inevitável (BEADLESS et al., 1979; HARVEY \& BRETHOUR, 1986; BRETHOUR et al., 1987; HALL \& DOISY, 1989; MOON et al., 1993). Entretanto, se por um lado, os diferentes princípios ativos utilizado ao longo dos anos contribuiram para o controle da mosca, certamente os diferentes métodos empregados aceleraram também os processos de químio-resistência da Haematobia (LOCKWOOD et al., 1985; QUISENBERRY et al., 1984; LUTHER \& BYFORD, 1987; BYFORD et al., 1987; SPARKS \& BYFORD, 1988, MACKINNON et al., 1991; CROSBY et al., 1991). Situações como estas podem ser evitadas em nossos criatórios, desde que seja usado o bom senso, e métodos racionais no controle da mosca.

O presente trabalho teve o objetivo de estudar o comportamento da $\boldsymbol{H}$. irritans em três propriedades rurais com manejo de controle parasitário habitual, sem contudo utilizar qualquer tratamento específico para a mosca.

\section{MATERIAIS E MÉTODOS}

a) Obtenção dos dados

No periodo de 1992 a 1994, foram feitas contagens da
H. irritans sobre a área dorso lombar de 30 bovinos mestiços europeu/zebu, em três propriedades agrícolas com criação extensiva, pertencentes à região de São Carlos, Estado de São Paulo. A região está situada a $234 \mathrm{~km}$ da capital do Estado, com clima classificado em Cwa, com inverno seco e as maiores precipitações pluviais acontecendo nos meses de verão (outubro a março), com regime hídrico údico. O solo predominante de Latossolo Vermelho-Amarelo, fase arenosa, faz parte da estrutura das fazendas estudadas com predominância na maioria, de pastagens de Brachiaria decumbens. Cada uma delas, localizada num município, possuia um manejo habitual, o qual correspondia ao tratamento parasitário $(\mathrm{T})$ destinado na propriedade:

$T_{1}$-Fazenda Ribeirão Bonito - verminose, tratado com levamizole-Ripercol L injetável; carrapato com piretróide "pour on" Ectoplus e berne com trichlorphonNeguvon + óleo queimado, uso tópico.

$\mathrm{T}_{2}$-Fazenda São Carlos, verminose tratada com levamizole-Ripercol L injetável; carrapatos com banho de imersão a base de formamidina- Triatox, e berne com fenthion-Tiguvon "pour on" $\mathrm{e}$

$\mathrm{T}_{3}$-Fazenda Santa Eudóxia, sem tratamento.

\section{b) Metodologia de análise}

Os dados de contagens da mosca-do-chifre, na escala $\sqrt{\mathrm{MC} \cdot 0,5}$ foram analisados segundo o modelo, considerando os efeitos de fazenda (F), ano (A), mês (M) conforme segue:

$$
\begin{aligned}
& Y_{i j k l}= \mu+F_{i}+A_{j}+(F A)_{i j}+M_{k}+(F M)_{j k}+(A M)_{i k}+\epsilon_{i j k l} \\
& \mathrm{i}= 1,2,3 ; \mathrm{j}=1,2,3 ; \mathrm{k}=1,2, \ldots, 12 ; \mathrm{l}=1,2, \ldots, \mathrm{n}_{\mathrm{ijk}} \\
& \mathrm{y}_{\mathrm{ijkl}}= \text { efeito do l-ésimo animal avaliado no k-ésimo } \\
& \text { mês do j-ésimo ano da i-ésima fazenda; } \\
& \mu= \text { efeito da média teórica; } \\
& \mathrm{F}_{\mathrm{i}}, \mathrm{A}_{\mathrm{j}}, \mathrm{M}_{\mathrm{k}}=\text { efeito principais de fazenda, ano e mês, } \\
& \text { respectivamente; } \\
&(\mathrm{FA})_{\mathrm{ij}},(\mathrm{FM})_{\mathrm{jk}},(\mathrm{AM})_{\mathrm{ik}}=\text { efeitos de interação; } \\
& \epsilon_{\mathrm{i} j \mathrm{kl}}=\text { erro aleatório suposto normalmente distribuido, } \\
& \text { com média zero e variância } \sigma^{2} .
\end{aligned}
$$

\section{RESULTADOS E DISCUSSÃO}

Os resultados obtidos, na escala $\sqrt{M C \cdot 0.5}$, considerando o comportamento da $\boldsymbol{H}$. irritans, foram distintos nos três municípios da região de São Carlos. $\mathrm{Na}$ Figura 1 estão apresentados os efeitos principais de fazenda (A), mês (B) e ano (C).Observa-se que as fazendas de São Carlos e de Ribeirão Bonito, apresentaram os menores níveis de infestação da mosca, 


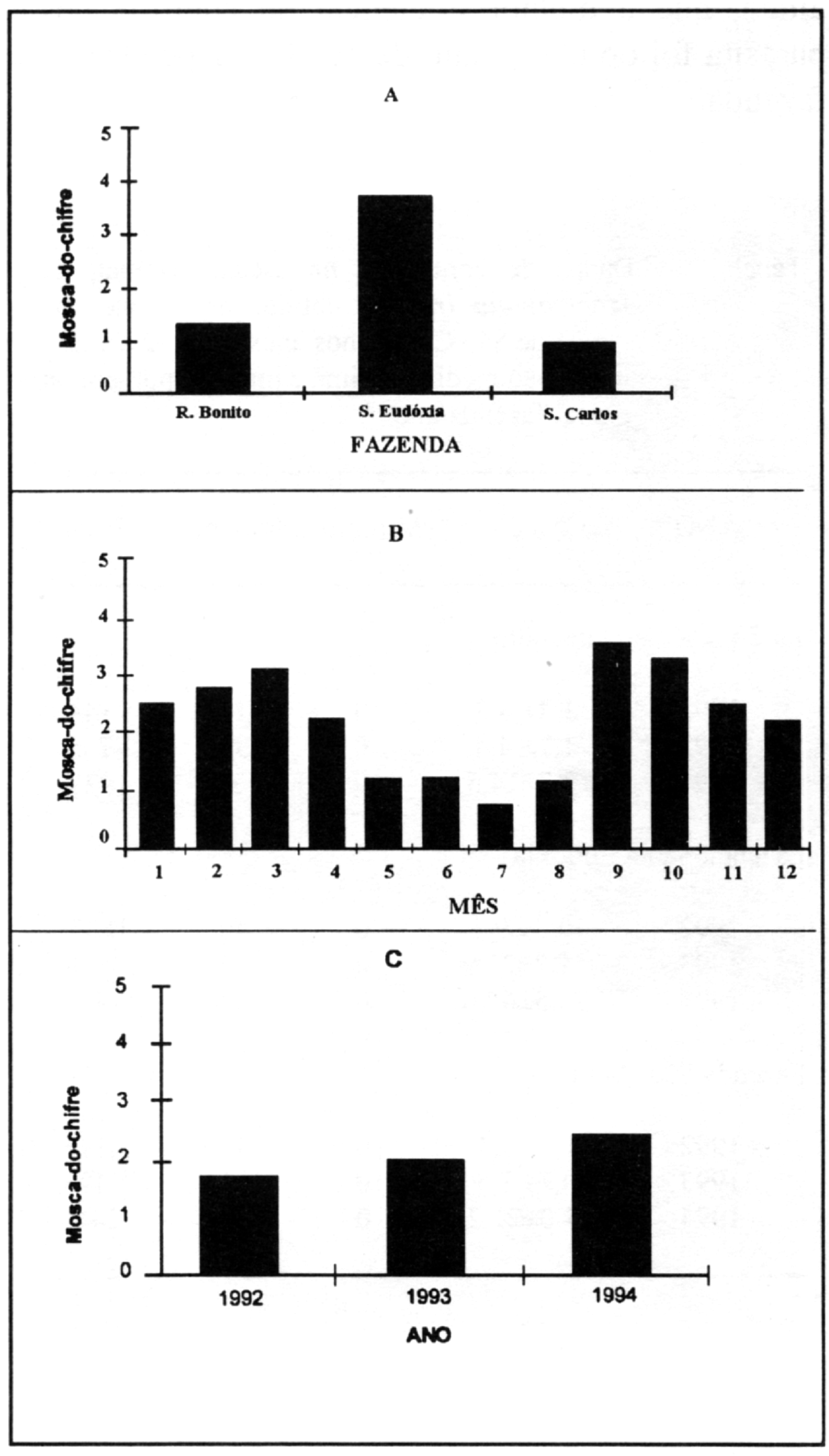

Figura 1 - Comportamento da Haematobia irritans (MC) em bovinos mestiços relativo a: fazendas (A), mês (B) e ano $(\mathrm{C})$, em três municípios da região de São Carlos, SP, nos anos de 1992, 1993 e 1994 (escala: $\sqrt{M C \cdot 0,5}$ ).

provavelmente em decorrência do tratamento utilizado. Independente da fazenda e do ano, verificou-se uma flutuação sazonal de mosca, ocorrendo maiores infestações nos meses mais quentes do ano (Figura 1 B).

Na Figura 2, estão apresentados os efeitos das interações $(P<0,0001)$ fazenda $x$ ano $(A)$, fazenda $\mathrm{x}$ mês (B) e ano $\mathrm{x}$ mês (C). A média de infestação/animal foi menor na fazenda de São Carlos $(1,1 \pm 1,0,0,9 \pm 0,6$ e 1,2 $\pm 1,7)$; enquanto que, na propriedade de Santa Eudóxia observou-se que a não utilização de tratamento parasítico induziu a liberdade de maior desenvolvimento da mosca-do-chifre $(2,9 \pm$ 1,5, 3,7 $\pm 3,0$ e 4,1 \pm 4,5), para os anos de 1992, 1993 e 1994, respectivamente. Contudo, observa-se que o nível de incidência da mosca nas propriedades de São
Carlos e Ribeirão Bonito, pode ser considerado dentro dos padrões de infestação aceitáveis da Haematobia.

A variação sazonal da mosca-do-chifre ao longo dos meses (Figura $2 \mathrm{~B}$ ), está relacionada com a fazenda, ou seja, sofreu a influência do tratamento habitual aplicado a cada uma delas; sendo observada incidência maior de mosca na fazenda de Santa Eudóxia, principalmente nos meses de temperaturas e precipitação pluvial mais elevadas, e população de mosca semelhante nas demais. Existem diferenças climáticas nos três municípios, porém, as linhas isotérmicas possuem tendências semelhantes, com temperaturas médias mais baixas no período de abril a setembro, acompanhado de estiagem no mesmo período (Figura 3). Independente de Fazenda, a incidência de mosca foi crescente nos anos de 1992 a 1994, principalmente nos meses de outubro a dezembro (Figura $2 \mathrm{C}$ ).

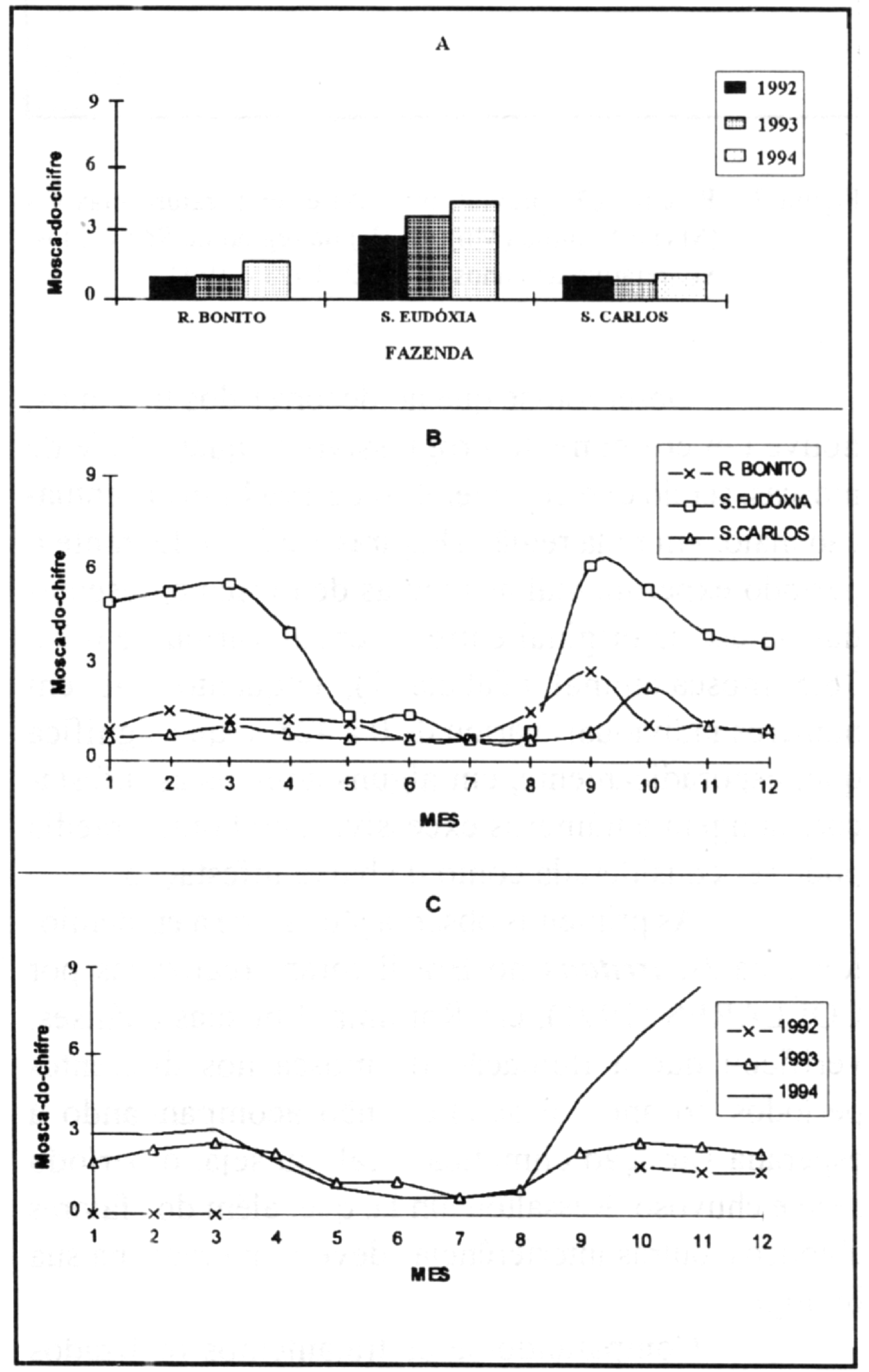

Figura 2 - Comportamento da Haematobia irritans (MC) em bovinos relativos a: fazenda $x$ ano $(A)$, fazenda $x$ mês (B) e ano $x$ mês (C), nos três municípios da região de São Carlos, SP, nos anos de 1992, 1993 e 1994 (escala: $\sqrt{M C \cdot 0,5}$ ). 


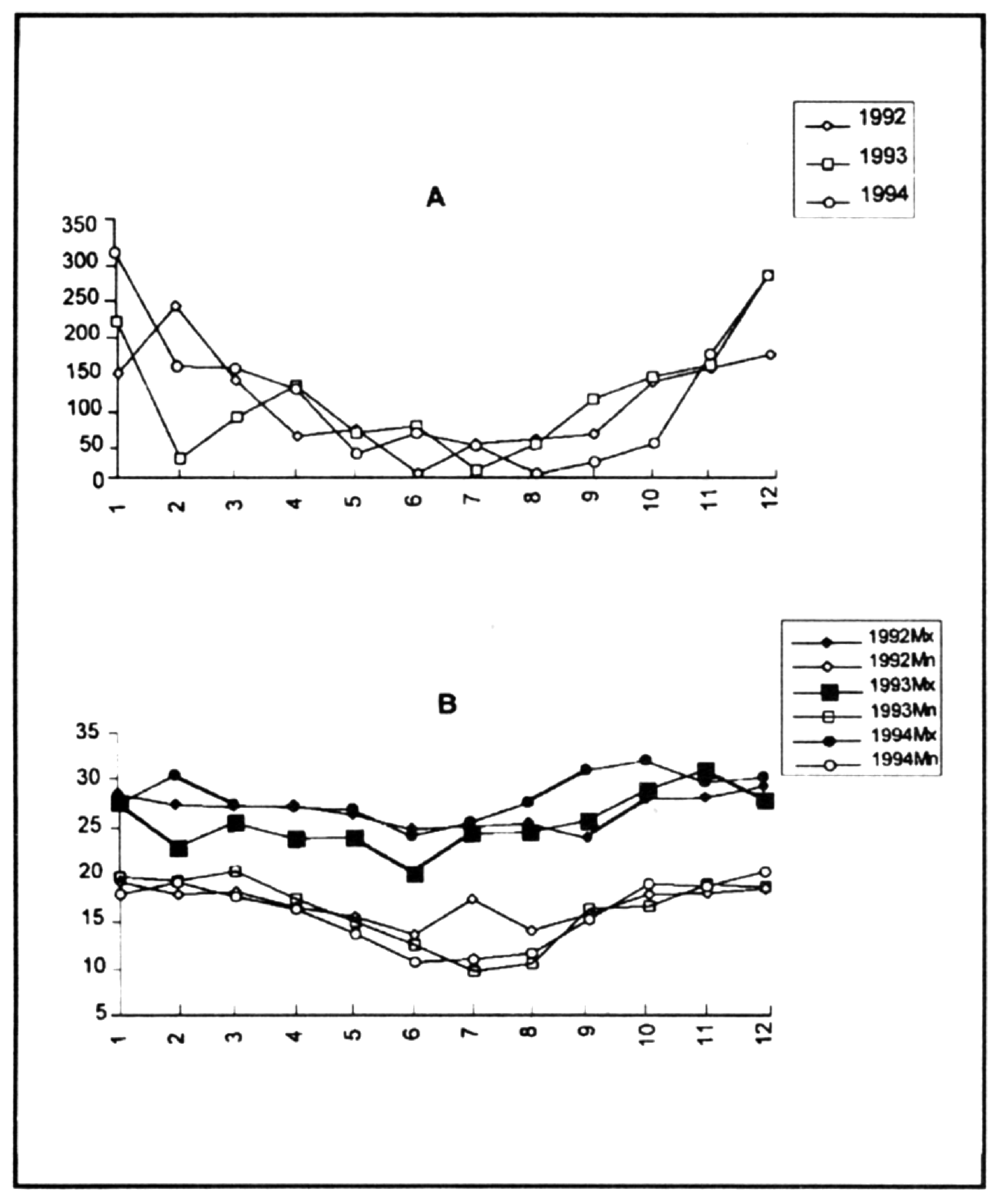

Figura 3 - Precipitação pluvial, mm (A) e temperatura máxima (Mx) e Mínima (Mn), ${ }^{0} \mathrm{C}(\mathrm{B})$ da região de São Carlos, SP, relativo aos anos de 1992, 1993 e 1994.

Observou-se que no decorrer dos três anos, houve um crescimento progressivo na quantidade de moscas, sendo que em setembro de 1994 uma acentuação maior em toda região (Figuras 1 e 2 C). Durante o período experimental as médias de mosca apresentadas na escala original entre fazendas variou de 0,7 a 36,5 mosca/animal (Tabela 1), enquanto que em números individuais variou de 0 a 428, o que significa que, esporadicamente, em alguns animais as infestações atingem a números excessivos, embora, a média pode ser considerada como de baixa infestação.

As primeiras observações sobre a epidemiologia da $\boldsymbol{H}$. irritans no Brasil foram realizadas por COLLARES (1991), em Roraima. Em suas análises, verificou que a flutuação da mosca nos diferentes períodos do ano foi acíclica, não acompanhando a esperada variação climática local, ou seja, o período seco e chuvoso. Ressaltou ainda, que, além dos fatores abióticos, outras interferências devem interagir na sua biologia.

Comparando-se os tratamentos realizados entre fazendas (Tabela 2), observou-se que a eficiência de controle das moscas variou de $81,2 \%$ (1992) a $92,0 \%$ (1993) em Ribeirão Bonito e de 66,6\% (1992) a 95,3\% (1993) em São Carlos, quando comparada ao tratamento controle (T3: Santa Eudóxia). Constatou-se ainda, que a melhor eficiência de controle desse parasita foi obtida no ano de 1993, independente de fazenda.

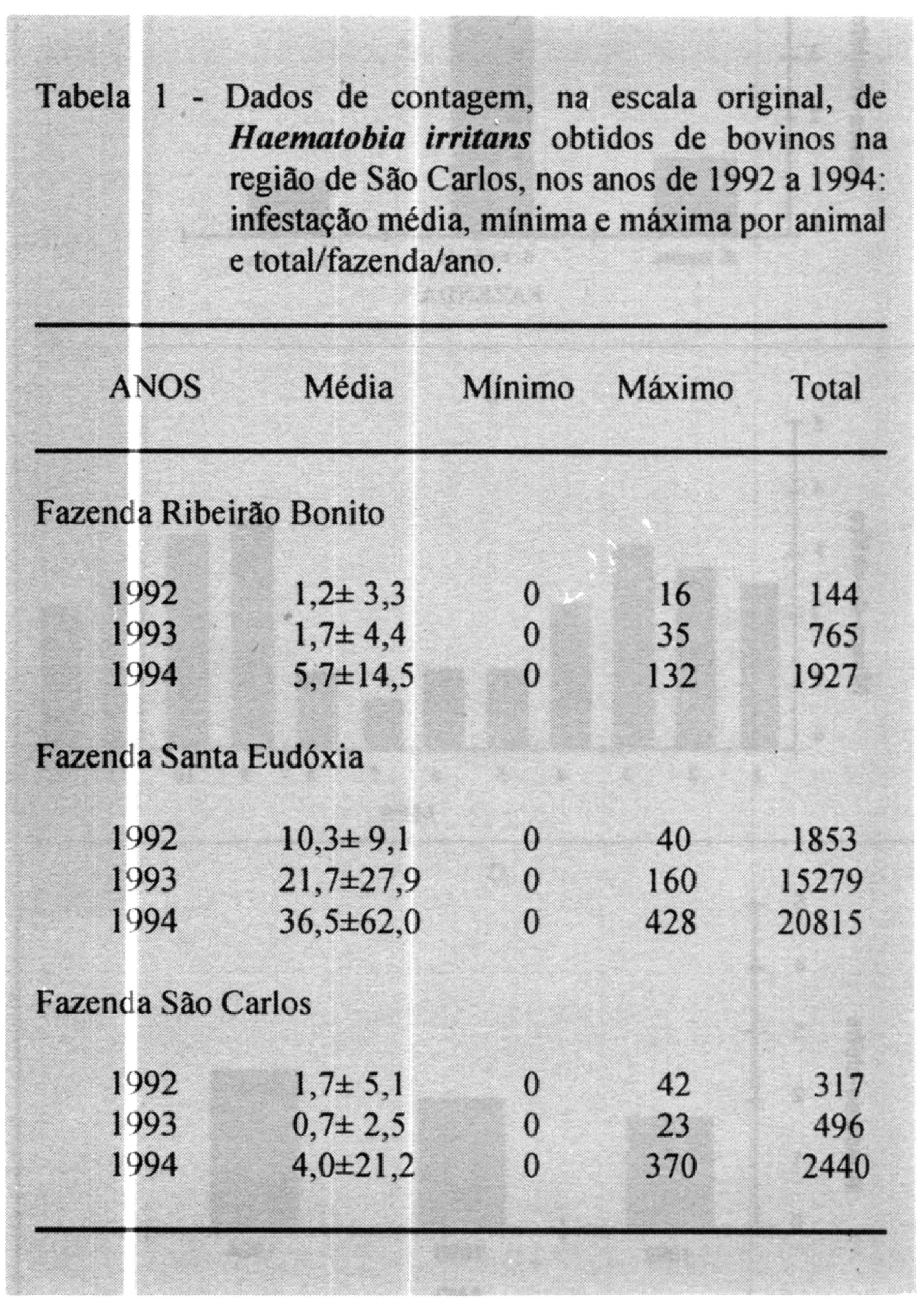

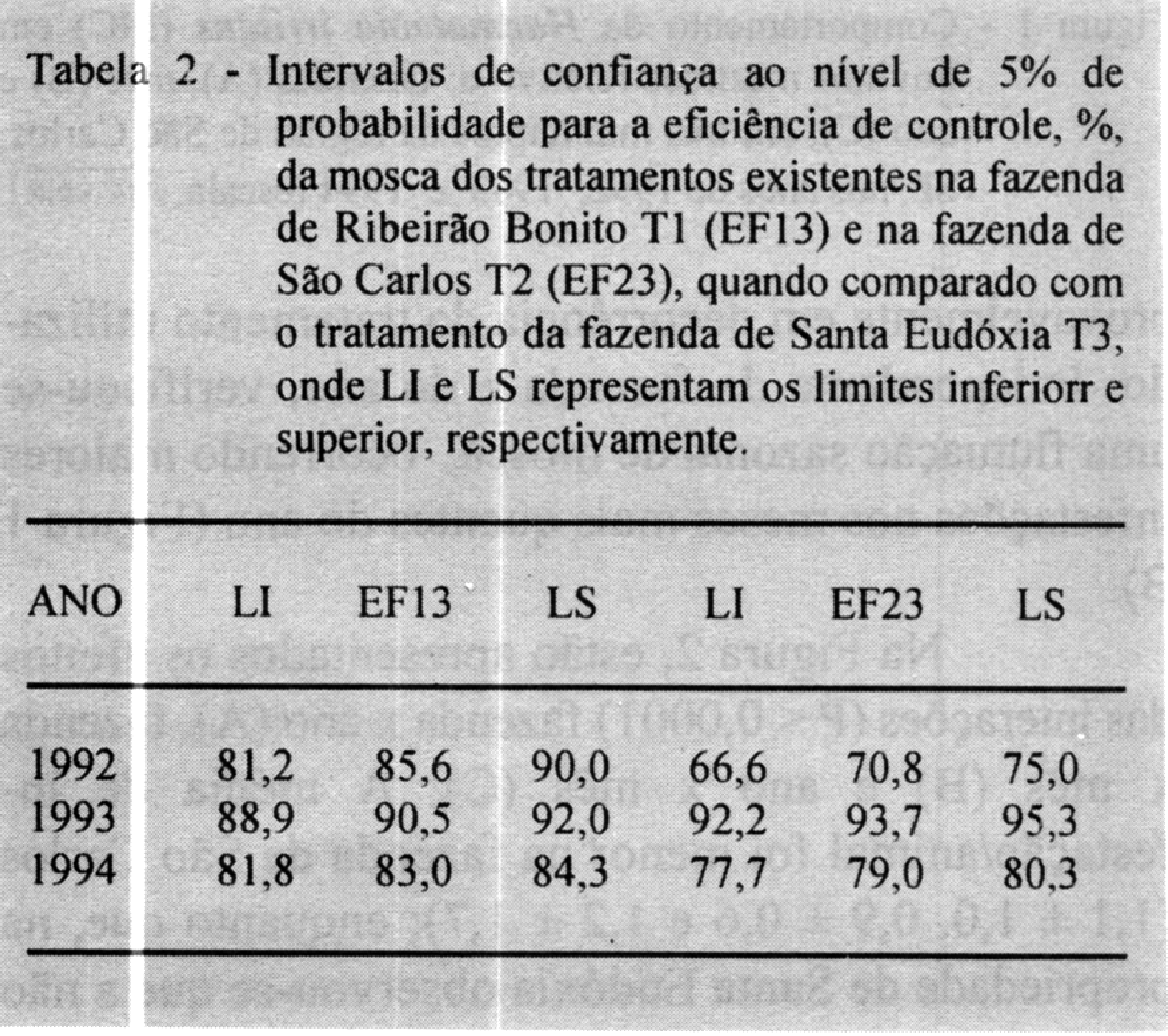

Em todas as análises realizadas, foram observados efeitos significativos nos resultados referentes à região de São Carlos, caracterizando que, 
em cada propriedade, provavelmente influenciada pelo manejo e pela localização, houve uma evolução diferenciada da mosca. GORDON et al. (1984) descreveram os fatores climáticos, temperaturas, precipitação pluvial e umidade, como intimamente relacionados ao desenvolvimento da mosca-do-chifre. No entanto, fundamentou, que, quando em excesso, esses elementos agem de forma negativa na sua biologia. Acrescentou ainda, que a localização da propriedade, tipos de explorações a que se destinam, tipos de solo, e "performance" da pastagem quanto ao período de crescimento, são fatores que predispõem a abundância da mosca-do-chifre.

Embora na região de São Carlos, em termos médios, a infestação da mosca apresentou-se relativamente baixa, considerando-se a média dos doze meses, alguns animais apresentaram infestações bem expressivas, ou seja, bem acima da média. Resultados semelhantes foram observados por BIANCHIN et al. (1993), os quais relataram que infestações médias de $\boldsymbol{H}$. irritans provenientes de dois períodos chuvosos no Estado de Mato Grosso do Sul não ultrapassavam a 80 moscas/animal. Na realidade, como relataram SAUERESSIG et al. (1995), as incidências de moscas/animal em nosso território, estão, em média, bem abaixo daquelas relatadas na literatura estrangeira (CAMPBELL, 1976; HARVEY \& BRETHOUR, 1986 e KUNZ et al., 1984). Portanto, as medidas de controle a serem adotadas devem ser criteriosas, e se possível adequando aos demais tratamentos ectoparasiticidas, evitando-se desta forma, ativar o processo de químioresistência antecipadamente. Como medida, toma-se como base a descrição de BURNS et al. 1975, em que preconizou o tratamento limiar de 200 moscas/animal, por considerar razoáveis as perdas econômicas. Verificando-se que em média, as infestações no rebanho dos municípios onde foram conduzidas as observações, estão bem abaixo deste nível, excetuando esporadicamente alguns animais, a medida a ser tomada será a utilização de controle somente nesses bovinos. Com isso, será favorecida a inter-relação entre gerações de moscas resistentes àquelas ainda sem resistência, atenuando-se, desta forma, o aparecimento de possíveis populações resistentes.

\section{CONCLUSÕES}

1) A eficiência dos tratamentos aplicados nas propriedades de Ribeirão Bonito e de São Carlos varia de $81,2 \%$ a $92,0 \%$ e $66,6 \%$ a $95,3 \%$, respectivamente; 2) O comportamento da mosca-do-chifre é atípico e acíclico entre os meses do ano e entres fazendas; 3) $\mathrm{Na}$ escala original, há uma carga de 15,7 $\pm 36,4$ moscas/animal nos meses de temperaturas mais elevadas (outubro a março) e de 6,8 $\pm 23,6$ moscas/animal nos meses de temperaturas mais baixas (abril a setembro); 4) Nos meses de estiagem e temperaturas mais baixas a incidência de moscas na região de São Carlos é reduzida.

\section{REFERÊNCIAS BIBLIOGRÁFICAS}

BEADLES, M.L., MILLER, J.A., SHELLEY, B.K., et al. Comparison of the efficacy of ear tags, leg bands, and tail tags for control of the horn fly on range cattle. The Southwestern Entomologist, v. 4, n. 1, p. 70-72, 1979.

BIANCHIN, I., HONER, M.R., KOLLER, W.W., et al. In: SEMINÁRIO BRASILEIRO DE PARASITOLOGIA VETERINÁRIA, 8, 1993. Londrina, PR. Anais... Londrina: CBPV, 1993 , p. 30.

BRETHOUR, J.R., HARVEY, .T.L., NEGUS, R., et al. Effect of cattle breed and flucythrinate-impregnated ear tags on horn fly (Diptera: Muscidae) control on yearling heifers. J Econ Entomol, v. 80, n. 5, p. 1035-1038, 1987.

BURNS, E.C., McCOY, G.R., MELACON, D.G., et al. Effect of horn flies on the rate of gain of stocker beef cattle. In: ANNUAL LIVESTOCK PRODUCERS DAY, 15., 1975. Proceeding... Baton Rouge, Lousiana State University, 1975, p. 258261

BYFORD, R., LOCKWOOD, J.A., SMITH, S.M., et al. Inseticide mixtures as an approach in the management of pyrethroidresistant hom flies (Dipetra: Muscidae) J Econ Entomol, v. 80, p. 111-116, 1987.

CAMPBELL, J.B. Effect of horn fly control on cows as expressed by increased weaning weights of calves. J Econ Entomol, v. 69, n. 6, p. $711-712,1976$.

COLLARES, N.C.P. Observaçð̃es epidemiológicas da Haematobia irritans em Roraima. In: SIMPÓSIO INTERNACIONAL SOBRE Haematobia irritans. I. 1991, São Paulo, SP. Anais.. São Paulo: CBPV, 1991. p. 20-25.

CROSBY, B.L., BYFORD, R.L., SPARKS, T.C. Bioassay for detecting active site insensitivity in horn fly (Diptera: Muscidae) larvae. J Econ Entomol, v. 84, n. 2, p. 367-370, 1991.

GORDON, D.V., HAYFE, W.O., KLEIN, K.K. Determination of economic thresholds for horn fly control in western Canada: A farm level simulation approach. Canadian J Agric Economics v. 32, n. 2 , p. $399-421,1984$.

HALL, R.D., DOISY, K.E. Walk-through trap for control of horn flies (Diptera: muscidae) on pastured cattle. J Econ Entomol v. 82 , n. 2 , p. $530-534,1989$.

HARVEY, T.L., BRETHOUR, J.R. Dust bag and backrubber applications of inseticides for control of pyrethroid resistant horn flies, Haematobia irritans (L). Preventive Veterinary Medicine. v. 3, p. 537-540, 1986

LOCKWOOD, J.A., BYFORD, R.L., STORY, R.N., et al. Behavioral resistance to the pyrethroids in the horn fly, Haematobia irritans (Diptera: muscidae). Environ Entomol, v. 14, p. 873 880,1985 
LUTHER, D.G., BYFORD, R.L. Ear tags control pyrethroid resistant horn flies on beef cattle. Louisiana Agriculture, v. 30. n. 3, p. 22-23, 1987.

MACKINNON, M.J., MEYER, R., HETZEL, D.J.S. Genetic variation and covariation for growth, parasite resistant and heat tolerance in tropical cattle. Livestock Prod Sci, v. 27, n. 2-3, p. 105-122, 1991 .

MOON, R.D., NOETZEL, D.M., JOHNSTON, L.J. Uptake and efficacy of methoprene and stirofos mineral blocks for control of horn flies (Diptera: muscidae) on pastured beef cattle. $\mathbf{J}$ Econ Entomol, v. 86, n. 6, p. 1738-1745, 1993.

QUISENBERRY, S.S., LOCKWOOD, J.A., BYFORD, R.L., et al.
Pyrethroid resistance in the horn fly, Haematobia irritans (L.) (Diptera: muscidae). J Econ Entomol, v. 77, n. 5, p. 10951098, 1984.

SAUERESSIG, T.M., SALVIANO, A., BARCELLOS, A.O., et al. Dinâmica populacional da mosca do chifre, Haematobia irritans e seu efeito no ganho de peso de bovinos na região do Distrito Federal. In: SEMINÁRIO BRASILEIRO DE PARASITOLOGIA VETERINÁIA, 9, 1995, Campo Grande, MS, Anais... Campo Grande: CBPV, 1995, p. 31.

SPARKS, T.C., BYFORD, R.L. Pyrethroid-synergist mixtures: toxicity, resistance, and field efficacy toward pyrethroidresistant horn flies (Diptera: muscidae). J Econ Entomol, v. 81, n. 6, p. $1567-1574,1988$.

Ciência Rural, v. 27, n. 2, 1997. 